Received: 6.11.2013.

Original scientific work

UDK:514.112

\title{
PARALLEL LINE SEGMENTS
}

Author: Antonio Polo

Italian High School in Rovinj (Croatia),

University “Juraj Dobrila” in Pula (Croatia)

email: toni.rovigno@gmail.com

\section{ABSTRACT}

In this article we will address some questions about a mathematical problem that Patrizio Frederic, a researcher in statistics at the University of Modena, proposed to the author. Given some parallel line segments, is there at least one straight line that passes through all of them? If there were many lines that solve the problem, can we choose a "best one" among all of them? We will fully address the first question. As for the second question, we will illustrate it with some "experimental" examples and suggest an outline for future explorations.

Keywords: Line segment, straight line, parallel, centroid, linear function, operator, point, Cartesian coordinate system, determinant, slope.

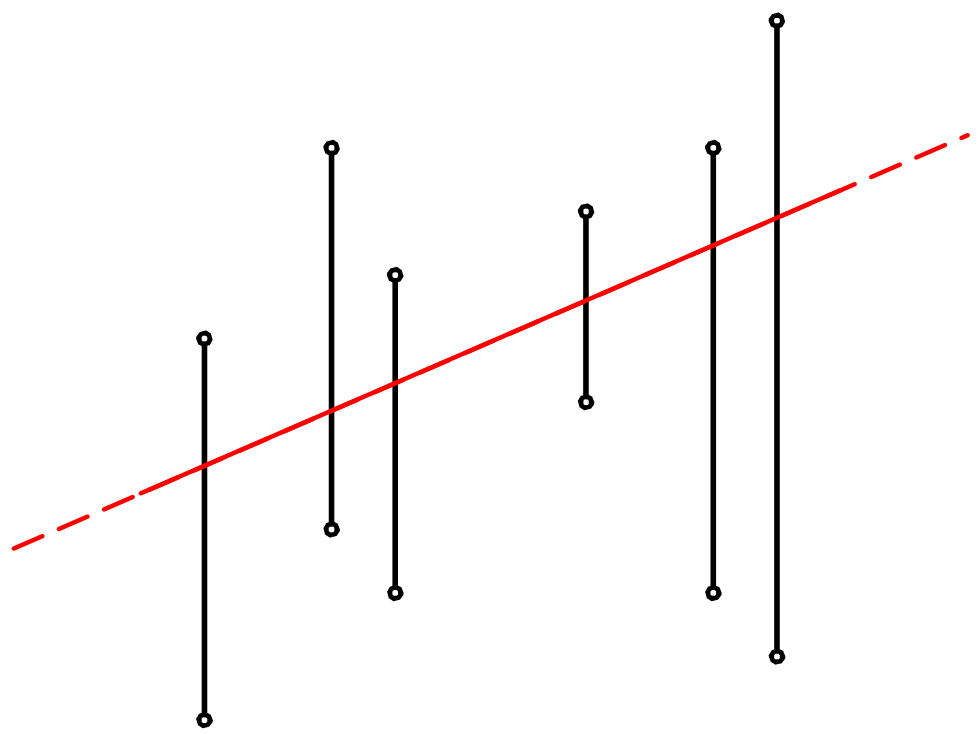




\section{INTRODUCTION}

When the problem has been formulated for the first time to us, the question was exactly this: "Do you know if there exists a quick way to determine whether we can intersect a series of parallel line segments with a straight line?". In this article, that we have divided into five sections, we have collected everything we have found on this subject. The first section includes this introduction. The second and the third are sections of technical nature in which we define the tools that we use, the real functional $\Phi$ and the space of real affine functions $\mathscr{L}\left(\mathbb{R}^{2}\right)$, and we prove their main properties. The fourth is the section in which we prove the main theorem, and we give a comprehensive answer to the question above. In the last chapter we studied some practical examples, applying the results obtained and also trying to give some hints to answer a question that has arisen in the meantime in our mind: "If the straight lines that solve the problem are many, with what criteria can we choose one of them saying that it is better than the others?".

\section{A PARTICULAR OPERATOR}

We begin by defining a tool that allows us to determine which are the respective positions of three points $A\left(x_{A}, y_{A}\right), B\left(x_{B}, y_{B}\right)$ and $C\left(x_{C}, y_{C}\right)$ on the Cartesian coordinate system. In particular we want to know if the three points are colinear or, alternatively, on what half-plane is one of the three points with respect to the straight line through the other two. This tool will be a functional defined by the determinant of a special square matrix of order 3.

For every given three points $A\left(x_{A}, y_{A}\right), B\left(x_{B}, y_{B}\right)$ and $C\left(x_{C}, y_{C}\right)$ on the Cartesian coordinate system, the functional $\Phi:\left(\mathbb{R}^{2}\right)^{3} \rightarrow \mathbb{R}$ is defined as follows:

$$
\Phi(A, B, C):=\operatorname{det}\left(\begin{array}{ccc}
1 & 1 & 1 \\
x_{A} & x_{B} & x_{C} \\
y_{A} & y_{B} & y_{C}
\end{array}\right) .
$$

We use the multilinearity of the determinant to prove some properties of the functional $\Phi$.

Proposition 1. $\Phi$ is invariant for translations.

Proof. Let $\mathcal{T}: \mathbb{R}^{2} \rightarrow \mathbb{R}^{2}$ be a translation given by a vector $\vec{v}=(a, b)$. We have that

$$
\Phi(\mathcal{T}(A), \mathcal{T}(B), \mathcal{T}(C))=\operatorname{det}\left(\begin{array}{ccc}
1 & 1 & 1 \\
x_{A}+a & x_{B}+a & x_{C}+a \\
y_{A}+b & y_{B}+b & y_{C}+b
\end{array}\right)=
$$




$$
\begin{gathered}
=\operatorname{det}\left(\begin{array}{ccc}
1 & 1 & 1 \\
x_{A} & x_{B} & x_{C} \\
y_{A}+b & y_{B}+b & y_{C}+b
\end{array}\right)+a \cdot \operatorname{det}\left(\begin{array}{ccc}
1 & 1 & 1 \\
1 & 1 & 1 \\
y_{A}+b & y_{B}+b & y_{C}+b
\end{array}\right) \\
=\operatorname{det}\left(\begin{array}{ccc}
1 & 1 & 1 \\
x_{A} & x_{B} & x_{C} \\
y_{A} & y_{B} & y_{C}
\end{array}\right)+b \cdot \overbrace{\operatorname{det}\left(\begin{array}{ccc}
1 & 1 & 1 \\
x_{A} & x_{B} & x_{C} \\
1 & 1 & 1
\end{array}\right)}^{=0}=\Phi(A, B, C) .
\end{gathered}
$$

Proposition 2. $\Phi$ is invariant for rotations.

Proof. Let $\mathcal{R}: \mathbb{R}^{2} \rightarrow \mathbb{R}^{2}$ be a rotation with rotation angle $\alpha$. We have that

$$
\begin{gathered}
\Phi(\mathcal{R}(A), \mathcal{R}(B), \mathcal{R}(C))=\operatorname{det}\left[\left(\begin{array}{ccc}
1 & 0 & 0 \\
0 & \cos \alpha & -\sin \alpha \\
0 & \sin \alpha & \cos \alpha
\end{array}\right) \cdot\left(\begin{array}{ccc}
1 & 1 & 1 \\
x_{A} & x_{B} & x_{C} \\
y_{A} & y_{B} & y_{C}
\end{array}\right)\right]= \\
=\overbrace{\operatorname{det}\left(\begin{array}{ccc}
1 & 0 & 0 \\
0 & \cos \alpha & -\sin \alpha \\
0 & \sin \alpha & \cos \alpha
\end{array}\right)}^{=1} \cdot \operatorname{det}\left(\begin{array}{ccc}
1 & 1 & 1 \\
x_{A} & x_{B} & x_{C} \\
y_{A} & y_{B} & y_{C}
\end{array}\right)=\Phi(A, B, C) .
\end{gathered}
$$

Proposition 3. Three points $A, B$ and $C$ on the Cartesian coordinate system are colinear iff $\Phi(A, B, C)=0$.

Proof. If two of the three points coincide then the proposition is obvious, there- fore we can assume that the three points are distinct. By the Proposition 2 is not restrictive to assume also that the points $A$ and $B$ are colinear with the $x$-axis, i.e. $y_{A}=y_{B}$. We have that

$$
\Phi(A, B, C)=\operatorname{det}\left(\begin{array}{ccc}
1 & 1 & 1 \\
x_{A} & x_{B} & x_{C} \\
y_{A} & y_{A} & y_{C}
\end{array}\right)=\left(x_{A}-x_{B}\right)\left(y_{A}-y_{C}\right)
$$

By the assumption that the points are distinct we have that $x_{A} \neq x_{B}$, then $\Phi(A, B, C)=0$ if $y_{A}=y_{C}$, i.e. if the points $A, B$ and $C$ are colinear.

Proposition 4. Let $A, B$ and $C$ be three points on the Cartesian plane, distinct and not colinear, and let $\mathscr{C}$ be the circle circumscribed to the triangle $A B C$, then $\Phi(A, B, C)>0$ if, along $\mathscr{C}$, the points $A, B$ and $C$ are ordered counterclockwise. Conversely $\Phi(A, B, C)<0$ if along $C$ the points $A, B$ and $C$ are ordered clockwise.

Proof. By the Proposition 1 it is not restrictive to assume that the circumcenter of the triangle $A B C$ coincides with the origin of the Cartesian plane and by the Proposition 2 it is not restrictive to assume that the points $A$ and $B$ are aligned with the $y$-axis, so that $x_{A}=x_{B}$ and $y_{A}<y_{B}$. Let $R$ be the radius of the circle circumscribed to the triangle $A B C$, then by the above assumptions, there are two angles $\alpha, \gamma$ satisfying $0<\alpha<\pi, 0 \leq \gamma<2 \pi, \gamma \neq \pm \alpha$ and such that 
$A(R \cos \alpha,-R \sin \alpha), B(R \cos \alpha, R \sin \alpha)$ and $C(R \cos \gamma, R \sin \gamma)$ are the Cartesian coordinates of the points $A, B$ and $C$.

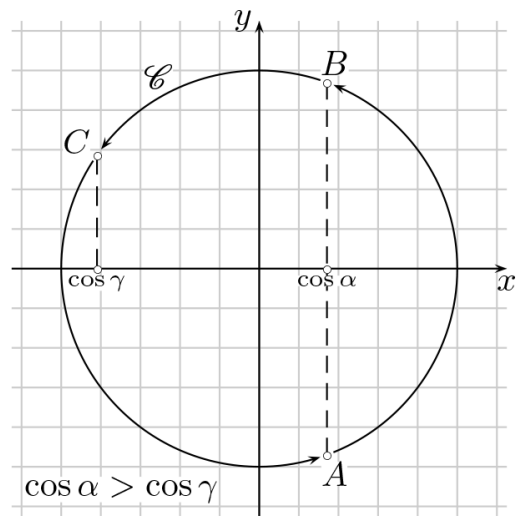

Figure 1.

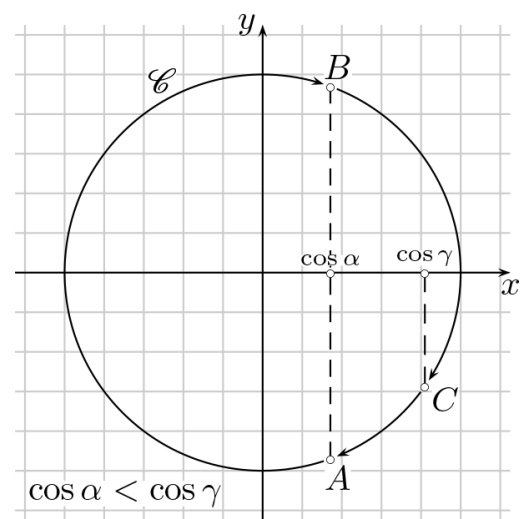

Figure 2.

In Figures 1 and 2 we highlight the fact that if the points $A, B$ and $C$ are ordered counterclockwise, then $\cos \alpha>\cos \gamma$, while if the points $A, B$ and $C$ are ordered clockwise, then $\cos \alpha$ $<\cos \gamma$. But by calculating $\Phi(A, B, C)$ we have that

$$
\Phi(A, B, C)=\operatorname{det}\left(\begin{array}{ccc}
1 & 1 & 1 \\
R \cos \alpha & R \cos \alpha & R \cos \gamma \\
-R \sin \alpha & R \sin \alpha & R \sin \gamma
\end{array}\right)=2 R^{2} \sin \alpha(\cos \alpha-\cos \gamma)
$$

and since $2 R^{2} \sin \alpha>0$, we conclude that:

$\Phi(A, B, C)>0 \Leftrightarrow \cos \alpha>\cos \gamma \Leftrightarrow$ the orientation is counterclockwise,

$\Phi(A, B, C)<0 \Leftrightarrow \cos \alpha<\cos \gamma \Leftrightarrow$ the orientation is clockwise.

To conclude this section, always regarding the functional $\Phi$, we make one final point that will be useful below.

Proposition 5. Let $A, B, C$ and $D$ be four points given on the Cartesian plane, positioned so that $x_{B}<x_{C}$ and $x_{A}=x_{D}$. The following conditions are equivalent:

$\begin{array}{ll}\text { i. } & y_{A} \geq y_{D}, \\ \text { ii. } & \Phi(A, B, C) \geq \Phi(D, B, C) \\ \text { iii. } & \Phi(B, A, C) \leq \Phi(B, D, C) \\ \text { iv. } & \Phi(B, C, A) \geq \Phi(B, C, D)\end{array}$

Moreover in all four cases equality holds iff $A \equiv D$. 
Proof. (i $\Leftrightarrow$ ii) We compute:

$$
\begin{gathered}
\Phi(A, B, C)-\Phi(D, B, C)=\operatorname{det}\left(\begin{array}{ccc}
1 & 1 & 1 \\
x_{A} & x_{B} & x_{C} \\
y_{A} & y_{A} & y_{C}
\end{array}\right)-\operatorname{det}\left(\begin{array}{ccc}
1 & 1 & 1 \\
x_{A} & x_{B} & x_{C} \\
y_{D} & y_{A} & y_{C}
\end{array}\right)= \\
=\operatorname{det}\left(\begin{array}{ccc}
0 & 1 & 1 \\
0 & x_{B} & x_{C} \\
y_{A}-y_{D} & y_{A} & y_{C}
\end{array}\right)=\left(y_{A}-y_{D}\right)\left(x_{C}-x_{B}\right),
\end{gathered}
$$

by assumption $x_{C}-x_{B}>0$, thus $\Phi(A, B, C) \geq \Phi(D, B, C) \Leftrightarrow y_{A} \geq y_{D}$, and moreover $\Phi(A, B, C)=\Phi(D, B, C) \Leftrightarrow y_{A}=y_{D} \Leftrightarrow \mathrm{A} \equiv \mathrm{D}$.

(ii $\Leftrightarrow \mathrm{iii} \Leftrightarrow \mathrm{iv}$ ) These equivalences follow from the fact that the determinant is alternating (or skew-symmetric) as well as multilinear.

\section{THE SPACE OF AFFINE FUNCTIONS}

Another tool we use is the space $\mathscr{L}\left(\mathbb{R}^{2}\right)$, wherein we represent the real affine functions, i.e. the functions $f: \mathbb{R} \rightarrow \mathbb{R}$ such that $f(x)=m x+b$ for some $(m, b) \in \mathbb{R}^{2}$.

The elements of $\mathscr{L}\left(\mathbb{R}^{2}\right)$ are in one-to-one correspondence with the straight lines on the Cartesian plane that are not parallel to the y-axis, these are in one-to-one correspondence with the equations of the form $y=m x+b$ and then, by the pair $(m, b)$, they are in one-to-one correspondence with the pairs of $\mathbb{R}^{2}$. This one-to-one correspondence has some interesting properties as shown in the next propositions.

In this section we use also the concept of "sheaf of straight lines", that is the set of straight lines all passing through the same point (except almost that parallel to the $y$-axis) or all parallel to each other.

Proposition 6. The set of all sheaves of straight lines on the Cartesian plane is in one-to-one correspondence with the set of straight lines of $\mathscr{L}\left(\mathbb{R}^{2}\right)$ and, in particular, a straight line in $\mathscr{L}\left(\mathbb{R}^{2}\right)$ is parallel to the $y$-axis if and only if its associated sheaf is composed by parallel straight lines.

Proof. Let $P\left(x_{P}, y_{P}\right) \in \mathbb{R}^{2}$ be a generic point, then the sheaf of straight lines passing through $P$ has explicit equation $y=m\left(x-x_{P}\right)+y_{P}$ and for all different $m \in \mathbb{R}$, in $\mathscr{L}\left(\mathbb{R}^{2}\right)$ the sheaf is represented by the locus of points whose coordinates are $\left(m,-x_{p} m\right.$ $\left.+y_{P}\right)$. This locus is a straight line whose explicit equation is $y=-x_{P} x+y_{P}$, then it is not parallel to the $y$-axis. However, if we have a sheaf of parallel straight lines in $\mathbb{R}^{2}$, we can express its equation in the form $y=k x+b$, where $b$ is a parameter and $k$ is constant. For all $b \in \mathbb{R}$ this sheaf is represented in $\mathscr{L}\left(\mathbb{R}^{2}\right)$ by the locus of points whose coordinates are $(k, b)$. Such a locus is a straight line, parallel to the $y$-axis, whose equation is $x=k$.

Proposition 7. The set of straight lines passing through a line segment parallel to the $y$-axis is convex in $\mathscr{L}\left(\mathbb{R}^{2}\right)$ and, in particular, it consists of the points of the plane between two parallel straight lines.

Proof. Let $\overline{A B}$ be a line segment parallel to the y-axis whose end-points are 
$A(a, b)$ and $B(a, c)$ (Figure 3$)$. A generic point $P_{t}$ of this segment has coordinates of type
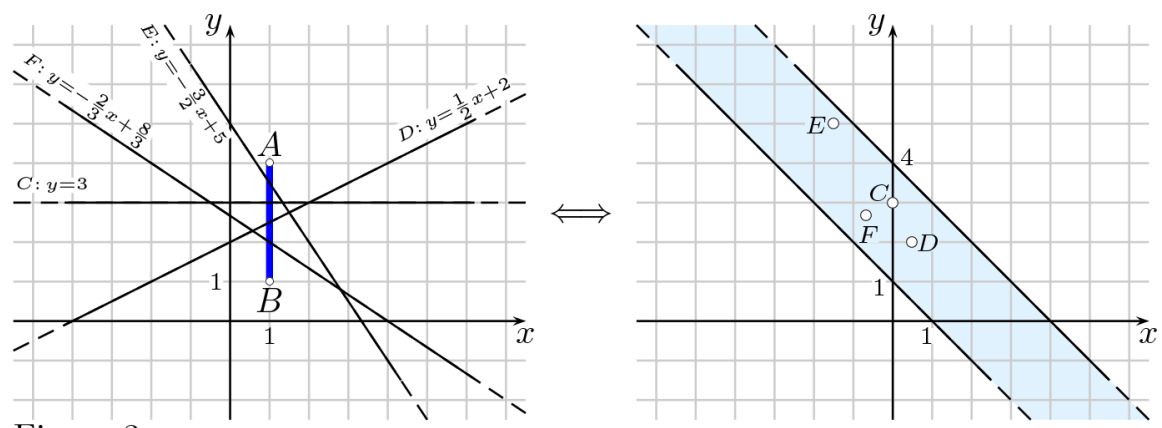

Figure 3.

$P_{t}(a, b+t(c-b))$ for some $t \in[0,1]$. As seen in the Proposition 6 , the sheaf of straight lines passing through $\mathrm{Pt}$ is represented in L (R2) by the straight lines with equation $y=$ $-a x+b+t(c-b)$, for all $t \in[0,1]$ this straight lines represent the set of all parallel lines between the lines $y=-a x+b$ and $y=-a x+c$ and then they form a convex set in $\mathscr{L}\left(\mathbb{R}^{2}\right)$.

Proposition 8. The set of straight lines passing through the points of two line segments parallel to the $y$-axis, but with distinct $x$-coordinate, is limited and convex in $\mathscr{L}\left(\mathbb{R}^{2}\right)$ and, in particular, it is a parallelogram whose centroid is associated with the straight line passing through the midpoints of the two line segments.

Proof. By the Proposition 7 it is clear that the set considered is a parallelogram. Then let $A(a, b), B(a, c), C(d, e)$ and $D(d, f)$ be the end-points of the two line segments and let $E\left(a, \frac{b+c}{2}\right)$ and $F\left(d, \frac{e+f}{2}\right)$ be the midpoints of $\overline{A B}$ and $\overline{C D}$, respectively. The equation of the straight line $E F$ will be

$$
y=\frac{b+c-e-f}{2(a-d)}(x-d)+\frac{e+f}{2}=\frac{b+c-e-f}{2(a-d)} x+\frac{a e+a f-b d-c d}{2(a-d)} .
$$

The equations of the straight lines $A C, A D, B C$ and $B D$ are:

$$
\begin{aligned}
& A C: y=\frac{b-e}{a-d} x+\frac{a e-b d}{a-d}, \quad A D: y=\frac{b-f}{a-d} x+\frac{a f-b d}{a-d} \text {, } \\
& B C: y=\frac{c-e}{a-d} x+\frac{a e-c d}{a-d} \text { and } B D: y=\frac{c-f}{a-d} x+\frac{a f-c d}{a-d} \text {. }
\end{aligned}
$$

These four straight lines, in $\mathscr{L}\left(\mathbb{R}^{2}\right)$, represent the vertices of the parallelogram corresponding to all straight lines passing through $\mathrm{AB}$ and $\mathrm{C} \mathrm{D}$. The centroid of this parallelogram is the midpoint of one of its diagonals, which is the point

$$
P\left(\frac{\frac{b-e}{a-d}+\frac{c-f}{a-d}}{2}, \frac{\frac{a e-b d}{a-d}+\frac{a f-c d}{a-d}}{2}\right) \Rightarrow P\left(\frac{b-e+c-f}{2(a-d)}, \frac{a e-b d+a f-c d}{2(a-d)}\right)
$$


whose coordinates, as we can see in (1), are exactly the slope and the y-intercept of the straight line $E F$.

\section{THE PROBLEM}

The problem we want to solve is the following: given a finite number, $n \geq 2$, of line segments which are closed, parallel, but mutually non-aligned, find equivalent conditions for having at least one straight line that intersects all segments.

\section{Notations (Figure 4).}

- Let the line segments be on a Cartesian coordinate system, so that they are parallel to the y-axis, and let's call them $L_{1}, L_{2}, \ldots, L_{n}$ in ascending order according to the $\mathrm{x}$-axis.

- Let $A_{i}\left(x_{i}, a_{i}\right)$ and $B_{i}\left(x_{i}, b_{i}\right)$ be the end-points of the $i$-th line segment, with $a_{i} \leq b_{i}$ for all $i \in\{1,2, \ldots, n\}$ and $x_{1}<x_{2}<\ldots<x_{n}$.

- Among all the straight lines passing through the end-points of the line segments we fix two particular ones: the straight line $A_{s} B_{t}$ whose slope $m_{s t}$ is the minimum between the slopes of all the straight lines $A_{i} B_{j}$ such that $1 \leq i<j \leq n$ and the straight line $B_{u} A_{v}$ whose slope $m_{u v}^{\prime}$ is the maximum between the slopes of all the straight lines $B_{i} A_{j}$ such that $1 \leq i<j \leq n$.

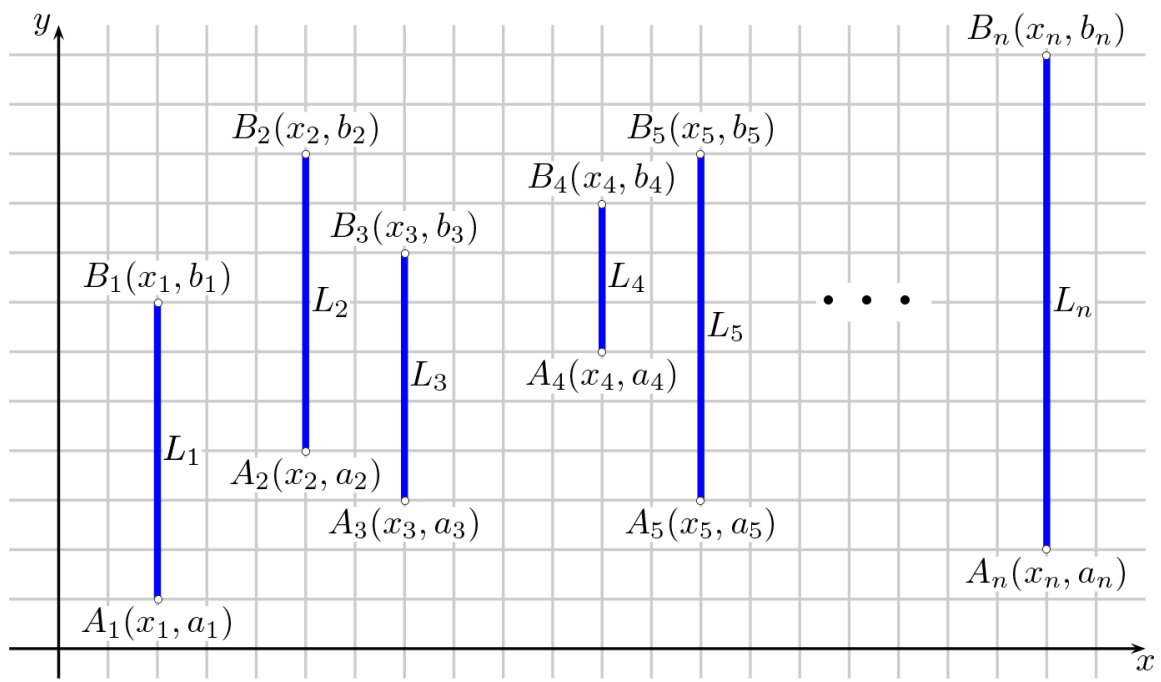

Figure 4. 
Theorem 9 (Main). With the previous notations and considering the nontrivial case $n \geq 3$, we have that the following statements are equivalent.

i. At least one straight line intersects all line segments $L_{1}, L_{2}, \ldots, L_{n}$.

ii. $\Phi\left(A_{i}, B_{j}, A_{k}\right) \leq 0 \leq \Phi\left(B_{i}, A_{j}, B_{k}\right)$ for all $i, j, k$ such that $1 \leq i<j<k \leq n$.

iii. $\Phi\left(A_{s}, B_{t}, A_{j}\right) \leq 0 \leq \Phi\left(B_{i}, A_{s}, B_{t}\right)$ for all $i, j$ such that $1 \leq i<s$ and $t<j \leq n$.

iv. $\Phi\left(A_{i}, B_{u}, A_{v}\right) \leq 0 \leq \Phi\left(B_{u}, A_{v}, B_{j}\right)$ for all $i, j$ such that $1 \leq i<u$ and $v<j \leq n$.

$v$. The straight line $A_{s} B_{t}$ intersects all line segments $L_{1}, L_{2}, \ldots, L_{n}$.

vi. The straight line $B_{u} A_{v}$ intersects all line segments $L_{1}, L_{2}, \ldots, L_{n}$.

Proof. (i $\Rightarrow$ ii) We fix three integers $i, j, k$ such that $1 \leq i<j<k \leq n$, then for assumption there exist three colinear points $P_{i}\left(x_{i}, y_{i}\right) \in L_{i}, P_{j}\left(x_{j}, y_{j}\right) \in \mathrm{L}_{j}$ and $P_{k}\left(x_{k}, y_{k}\right) \in$ $L_{k}$ such that $a_{p} \leq y_{p} \leq b_{p}$ for all $p \in\{i, j, k\}$. Then by the Propositions 3 and 5 we have that

$$
\begin{gathered}
\Phi\left(A_{i}, B_{j}, A_{k}\right) \leq \Phi\left(P_{i}, B_{j}, A_{k}\right) \leq \Phi\left(P_{i}, P_{j}, A_{k}\right) \leq \overbrace{\Phi\left(P_{i}, P_{j}, P_{k}\right)}^{=0} \leq \\
\leq \Phi\left(B_{i}, P_{j}, P_{k}\right) \leq \Phi\left(B_{i}, A_{j}, P_{k}\right) \leq \Phi\left(B_{i}, A_{j}, B_{k}\right) .
\end{gathered}
$$

(ii $\Rightarrow$ iii), (ii $\Rightarrow$ iv) Obvious.

(iii $\Rightarrow \mathrm{v}$ ) For all $i \in\{1,2, \ldots, n\}$ let $P_{i}\left(x_{i}, y_{i}\right)$ be the intersection point between the straight lines $A_{s} B_{t}$ and $x=x_{i}$. We analyze four cases.

1st case: $1 \leq i<s$. For assumption $\Phi\left(B_{i}, A_{s}, B_{t}\right) \geq 0$ and by the Proposition 3 $\Phi\left(P_{i}, A_{s}, B_{t}\right)=0$, then by the Proposition 5 we have that $b_{i} \geq y_{i}$.

2nd case: $1 \leq i<t$. We know that $m_{s t} \leq m_{i t}$ and that the point $P_{i}$ belongs to the straight line $A_{s} B_{t}$, then we have that

$$
m_{s t} \leq m_{i t} \quad \Leftrightarrow \quad \frac{y_{i}-b_{t}}{x_{i}-x_{t}} \leq \frac{a_{i}-b_{t}}{x_{i}-x_{t}} \quad \Leftrightarrow \quad y_{i}-b_{t} \geq a_{i}-b_{t} \quad \Leftrightarrow \quad y_{i} \geq a_{i} .
$$

3rd case: $s<i \leq n$. We know that $m_{s t} \leq m_{i t}$ and that the point $P$ belongs to the straight line $A_{s} B_{t}$, then we have that

$$
m_{s t} \leq m_{s i} \quad \Leftrightarrow \quad \frac{a_{s}-y_{i}}{x_{s}-x_{i}} \leq \frac{a_{s}-b_{i}}{x_{s}-x_{i}} \quad \Leftrightarrow \quad a_{s}-y_{i} \geq a_{s}-b_{i} \quad \Leftrightarrow \quad y_{i} \leq b_{i} .
$$

4th case: $t<i \leq n$. By the assumption $\Phi\left(A_{s}, B_{t}, A_{i}\right) \leq 0$ and by the Proposition $3 \Phi\left(A_{s}, B_{t}, P_{i}\right.$ ) $=0$, then by the Proposition 5 we have that $a_{i} \leq y_{i}$.

Adding the two trivial inequalities $b_{s} \geq y_{s}=a_{s}$ and $a_{t} \leq y_{t}=b_{t}$, in the four cases are proved the inequalities $a_{i} \leq y_{i} \leq b_{i}$ for all $i \in\{1,2, \ldots n\}$, then the stright line $A_{s} B_{t}$ intersects all $n$ line segments.

(iv $\Rightarrow$ vi) Quite similar to the previous proof.

( $v \Rightarrow \mathrm{i})$, (vi $\Rightarrow$ i) Obvious. 
Corollary 10. The following statements are equivalent.

i. There is one and only one straight line that intersects all the line segments $L_{1}, L_{2}, \ldots$, $L_{n}$.

ii. The two straight lines $A_{s} B_{t}$ and $B_{u} A_{v}$ coincide and intersect all the line segments $L_{1}, L_{2}$ $, \ldots, L_{n}$.

iii. $\Phi\left(A_{i}, B_{j}, A_{k}\right) \leq 0 \leq \Phi\left(B_{i}, A_{j}, B_{k}\right)$ for all $i, j, k$ such that $1 \leq i<j<k \leq n$ and, if we are not in presence of the limit case in which two of the segments degenerate into a point, then at least in one case there is the equality

$$
\Phi\left(A_{i}, B_{j}, A_{k}\right)=0 \text { or } \Phi\left(B_{i}, A_{j}, B_{k}\right)=0 .
$$

Proof. (i $\Rightarrow$ ii) Obvious by the equivalence $\mathrm{i} \Leftrightarrow$ iii $\Leftrightarrow$ iv in the Theorem 9 .

(ii $\Leftrightarrow$ iii) By the Theorem 9 we are sure that $\Phi\left(A_{i}, B_{j}, A_{k}\right) \leq 0 \leq \Phi\left(B_{i}, A_{j}, B_{k}\right)$

for all $i, j, k$ such that $1 \leq i<j<k \leq n$. The four points $A_{s}, B_{t}, B_{u}$ and $A_{v}$ are, for assumption, colinear; assuming that there are not two segments that degenerate to a point, then it can not happen simultaneously that $A_{s} \equiv B_{u}$ and $B_{t} \equiv A_{v}$, then we are sure that in addition with the already known inequalities $x_{s}<x_{t}$ and $x_{u}<x_{v}$, also at least one of these occurs:

$$
x_{s}<x_{u}, \quad x_{u}<x_{s}, \quad x_{t}<x_{v} \quad x_{v}<x_{t} .
$$

So, in each of the four cases we have that

$$
\begin{array}{lll}
x_{s}<x_{u} \text { and } & x_{u}<x_{v} \Rightarrow & \Phi\left(A_{s}, B_{u}, A_{v}\right)=0, \\
x_{u}<x_{s} \text { and } & x_{s}<x_{t} \Rightarrow & \Phi\left(B_{u}, A_{s}, B_{t}\right)=0, \\
x_{t}<x_{v} \text { and } & x_{s}<x_{t} \Rightarrow & \Phi\left(A_{s}, B_{t}, A_{v}\right)=0, \\
x_{v}<x_{t} \text { and } & x_{u}<x_{v} \Rightarrow & \Phi\left(B_{u}, A_{v}, B_{t}\right)=0 .
\end{array}
$$

(iii $\Rightarrow$ i) By the Theorem 9 at least one straight line exists, the uniqueness would be obvious if two of the line segments were pointlike, so we assume that $\Phi\left(A_{i}, B_{j}, A_{k}\right)=0$ for some $\mathrm{i}, \mathrm{j}, \mathrm{k}$ such that $1 \leq i<j<k \leq n$ (the proof is similar if we assume that $\left.\Phi\left(B_{i}, A_{j}, B_{k}\right)=0\right)$. A straight line that crosses the segments $L_{i}, L_{j}$ and $L_{k}$ will pass through the three colinear points $P_{i} \in L_{i}, P_{j} \in L_{j}$ and $P_{k} \in L_{k}$, then by the Proposition 5 we have that

$$
0=\Phi\left(A_{i}, B_{j}, A_{k}\right) \leq \Phi\left(P_{i}, B_{i}, A_{k}\right) \leq \Phi\left(P_{i}, P_{j}, A_{k}\right) \leq \Phi\left(P_{i}, P_{j}, P_{k}\right)=0 .
$$

Since all inequalities are equalities then, once again by the Proposition 5 , we have that $P_{i} \equiv A_{i}, P_{j} \equiv B_{j}$ and $P_{k} \equiv A_{k}$ and the straight line can only be unique.

Corollary 11. Among all the straight lines that pass through the line segments $L_{1}, L_{2}, \ldots, L_{n}$ (assuming that there exists any), the straight lines $A_{s} B_{t}$ and $B_{u} A_{v}$ are those with maximum and minimum slope respectively. 
Proof. We consider a line $r$ with slope $m>m_{s t}$, that passes through the points $P_{s}\left(x_{s}, y_{s}\right) \in L_{s}$ and $P_{t}\left(x_{t}, y_{t}\right)$ and we prove that $P_{t} \notin L_{t}$. We calculate:

$$
\begin{gathered}
m_{s t}<m \Rightarrow \frac{a_{s}-b_{t}}{x_{s}-x_{t}}<\frac{y_{s}-y_{t}}{x_{s}-x_{t}} \Rightarrow \\
\Rightarrow \quad a_{s}-b_{t}>y_{s}-y_{t} \Rightarrow y_{t}-b_{t}>y_{s}-a_{s} \geq 0 .
\end{gathered}
$$

Since $y_{t}>b_{t}$ then the straight line $r$ does not cross the line segment $L_{t}$ and this proves that $m_{s t}$ is the maximum slope between those of the straight lines that cross all segments. Similarly $m_{u v}^{\prime}$ is the minimum of the slopes.

\section{A SPECIAL STRAIGHT LINE}

Now we are able to suggest some algorithms that allow us to find a straight line, among others, that can cross in the "best way" all line segments.

\section{Algorithm 1. A (not so) special straight line (Figure 5 and 6).}

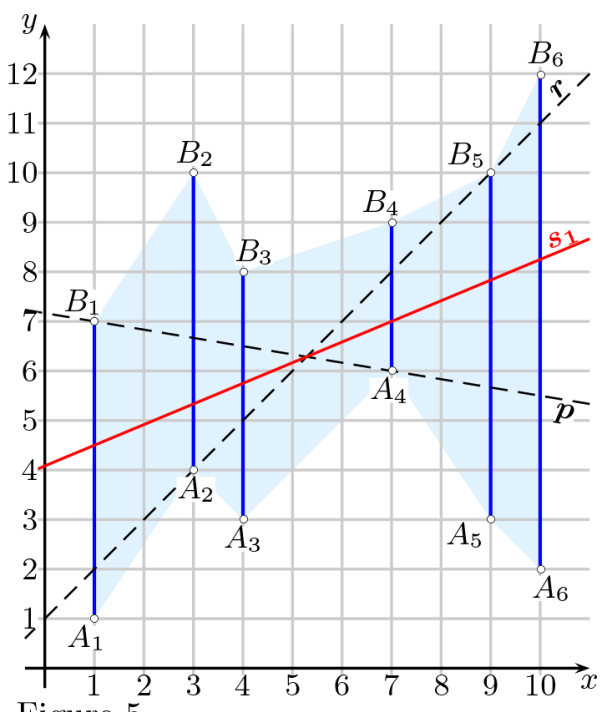

Figure 5 .

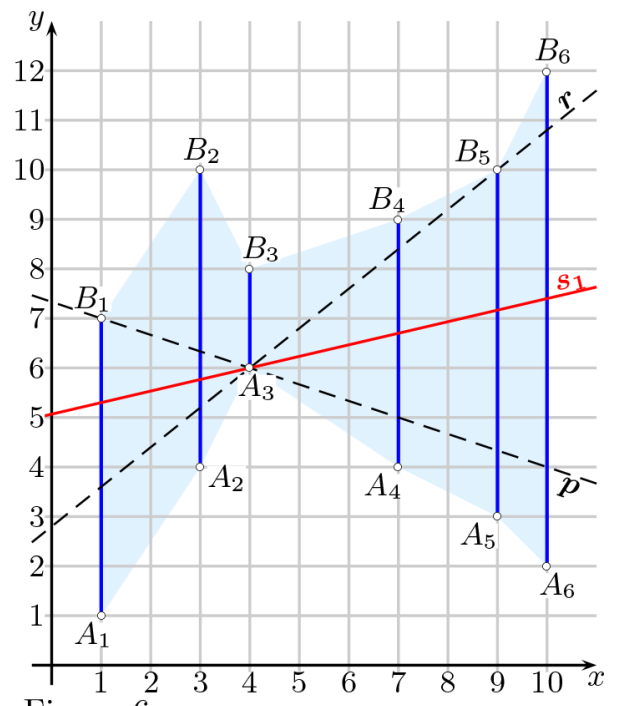

Figure 6.

1. Let the $n$ line segments be given, then we find the straight line $r=A_{s} B_{t}$.

2. We check if $\Phi\left(B_{i}, A_{s}, B_{t}\right) \geq 0$ for all $i \in\{1,2, \ldots, s-1\}$.

3. We check if $\Phi\left(A_{s}, B_{t}, A_{j}\right) \leq 0$ for all $\mathrm{j} \in\{t+1, t+2, \ldots, n\}$.

4. If the checks at points 2 and 3 have failed then there is no reason to continue, otherwise we can find also the straight line $\mathrm{p}=\mathrm{Bu} \mathrm{Av}$. By the Theorem 9 we are sure that the straight lines $\mathrm{r}$ and $\mathrm{p}$ pass through all the line segments $L_{1}, L_{2}, \ldots, L_{n}$. 
5. We choose the line $s_{1}$ that, in $\mathscr{L}\left(\mathbb{R}^{2}\right)$, corresponds to the midpoint between $r$ and $p$. By the Proposition 7 the set of all the straight lines passing through all of the line segments $L_{1}, L_{2}, \ldots, L_{n}$ is convex, then $s_{1}$ also passes for all of those line segments.

In Figure 5 we have that

$$
r: y=x+1 \quad \text { and } \quad p: y=-\frac{1}{6} x+\frac{43}{6} \quad \Rightarrow \quad s_{1}: y=\frac{5}{12} x+\frac{49}{12} ;
$$

in Figure 6 we have that

$$
r: y=\frac{4}{5} x+\frac{14}{5} \quad \text { and } \quad p: y=-\frac{1}{3} x+\frac{22}{3} \quad \Rightarrow \quad s_{1}: y=\frac{7}{30} x+\frac{76}{15} .
$$

But this algorithm does not always give us a "good" straight line. As it is shown in Figure 6, sometimes the straight line we get does not seem to be one of the best and in this example the straight line passes through the end-point of one of the line segments.

\section{Algorithm 2. A straight line a little bit more special.}

1. We perform steps $1,2,3$ and 4 of Algorithm 1 .

2. Among all the straight lines $A_{i} A_{j}$, with $1 \leq i<j \leq n$, we take only those that cross all line segments $L_{1}, L_{2}, \ldots, L_{n}$, that are distinct from $r$ and $p$ and we take only once if two or more of them are identical.

3. Among all the straight lines $B_{i} B_{j}$, with $1 \leq i<j \leq n$, we take only those that cross all line segments $L_{1}, L_{2}, \ldots, L_{n}$, that are distinct from $r$ and $p$ and we take only once if two or more of them are identical.

4. By the Proposition 7 all the straight lines found in steps 2 and 3, together with $r$ and $p$, are the vertices of a convex polygon in $\mathscr{L}\left(\mathbb{R}^{2}\right)$. Let us take the straight line $s_{2}$ that corresponds to the centroid of the vertices of this polygon.

Let us review the example in Figure 5. 


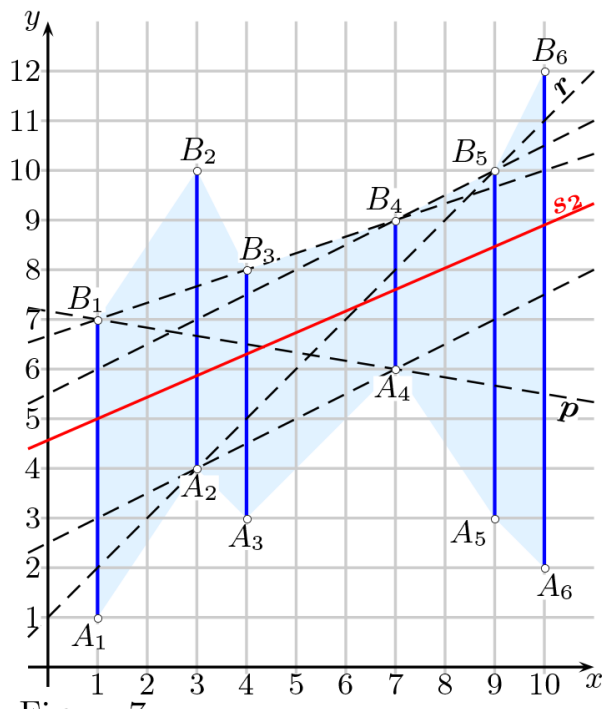

Figure 7 .

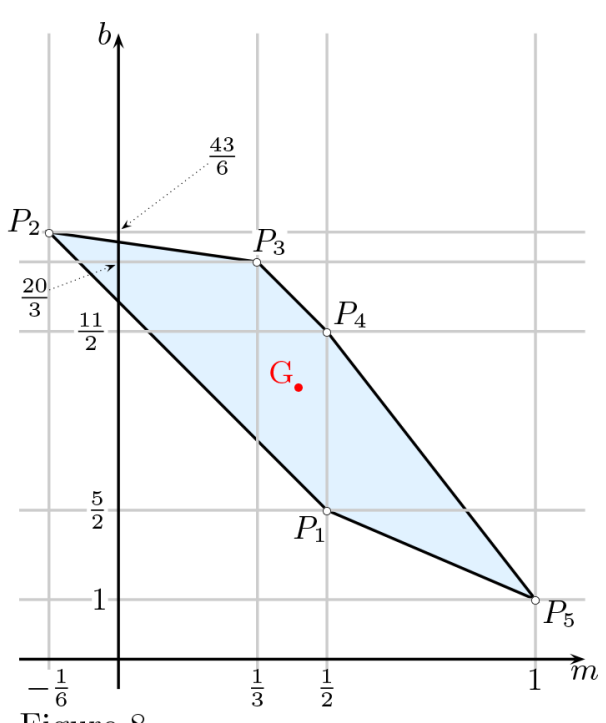

Figure 8.

In Figure 7 we have represented the line segments and in Figure 8 we have represented, in $\mathscr{L}\left(\mathbb{R}^{2}\right)$, the convex polygon formed by all the straight lines that cross all line segments; the point $G$ is the centroid of the vertices of the polygon and it is associated with the straight line $s_{2}$ that would seem to go through the line segments better than the straight line $s_{1}$ in Figure 5. In summary we have:

$$
\begin{gathered}
A_{4} B_{1}: y=-\frac{1}{6} x+\frac{43}{6} \Rightarrow P_{2}\left(-\frac{1}{6}, \frac{43}{6}\right), A_{2} A_{4}: y=\frac{1}{2} x+\frac{5}{2} \Rightarrow P_{1}\left(\frac{1}{2}, \frac{5}{2}\right), \\
B_{1} B_{4}: y=\frac{1}{3} x+\frac{20}{3} \Rightarrow P_{3}\left(\frac{1}{3}, \frac{20}{3}\right), \quad B_{5} A_{2}: y=x+1 \Rightarrow P_{5}(1,1), \\
B_{4} B_{5}: y=\frac{1}{2} x+\frac{11}{2} \quad \Rightarrow P_{4}\left(\frac{1}{2}, \frac{11}{2}\right) ; \\
m_{G}=\frac{-\frac{1}{6}+\frac{1}{3}+\frac{1}{2}+\frac{1}{2}+1}{5}=\frac{13}{30}, \quad b_{G}=\frac{\frac{43}{6}+\frac{20}{3}+\frac{11}{2}+\frac{5}{2}+1}{5}=\frac{137}{30}
\end{gathered}
$$

and finally the equation of $s_{2}$ is $y=\frac{13}{30} x+\frac{137}{30}$.

Let us review also the example in Figure 6. 


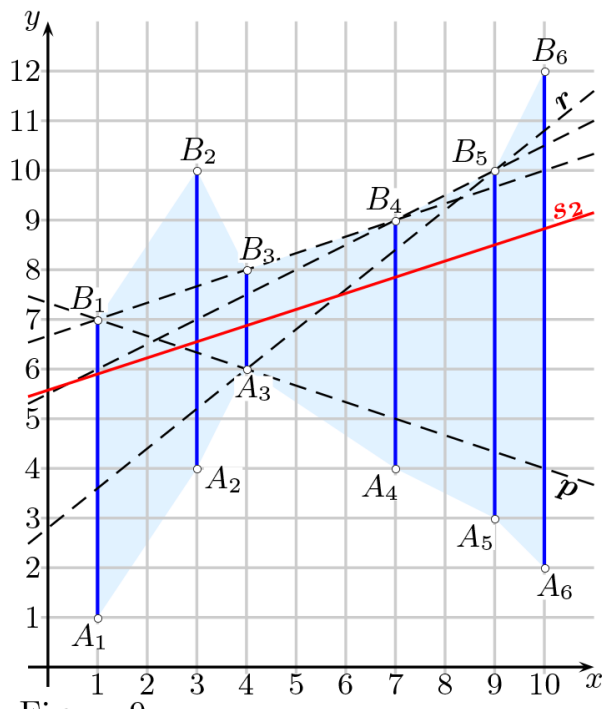

Figure 9.

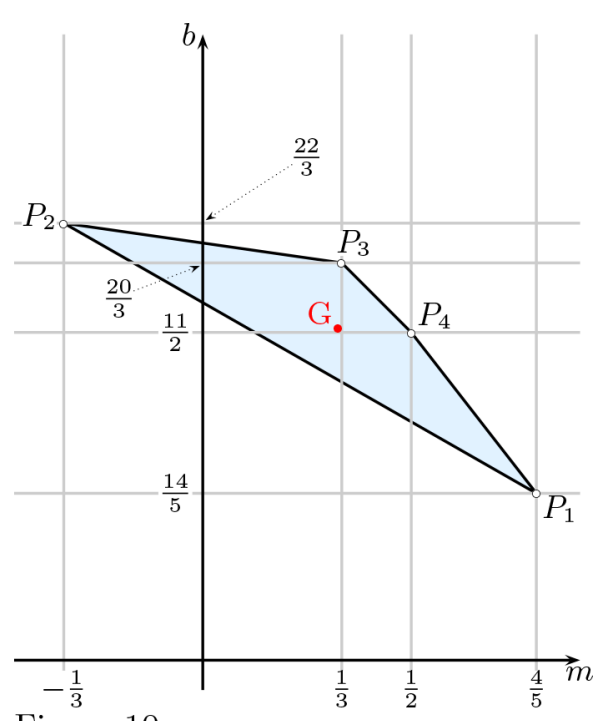

Figure 10.

Even in this example the straight line $s_{2}$ crosses all the line segments in a very "better" way than the straight line $s_{1}$ do in Figure 6. In summary in this example we have:

$$
\begin{gathered}
B_{5} A_{3}: y=\frac{4}{5} x+\frac{14}{5} \Rightarrow P_{1}\left(\frac{4}{5}, \frac{14}{5}\right), A_{3} B_{1}: y=-\frac{1}{3} x+\frac{22}{3} \Rightarrow P_{2}\left(-\frac{1}{3}, \frac{22}{3}\right), \\
B_{1} B_{4}: y=\frac{1}{3} x+\frac{20}{3} \Rightarrow P_{3}\left(\frac{1}{3}, \frac{20}{3}\right), B_{4} B_{5}: y=\frac{1}{2} x+\frac{11}{2} \Rightarrow P_{4}\left(\frac{1}{2}, \frac{11}{2}\right) ; \\
m_{G}=\frac{\frac{4}{5}-\frac{1}{3}+\frac{1}{3}+\frac{1}{2}}{4}=\frac{13}{40}, \quad b_{G}=\frac{\frac{14}{5}+\frac{22}{3}+\frac{20}{3}+\frac{11}{2}}{4}=\frac{223}{40}
\end{gathered}
$$

and finally $s_{2}: y=\frac{13}{40} x+\frac{223}{40}$.

\section{Algorithm 3. An even more special straight line.}

1. We perform steps 1, 2 and 3 of Algorithm 2 .

2. We do the same observations done in the step 4 of the Algorithm 2, but in this case, as straight line $s_{3}$, we take the straight line associated with the centroid of the whole polygon that we find in $\mathscr{L}\left(\mathbb{R}^{2}\right)$.

Let us review again the two examples above. 


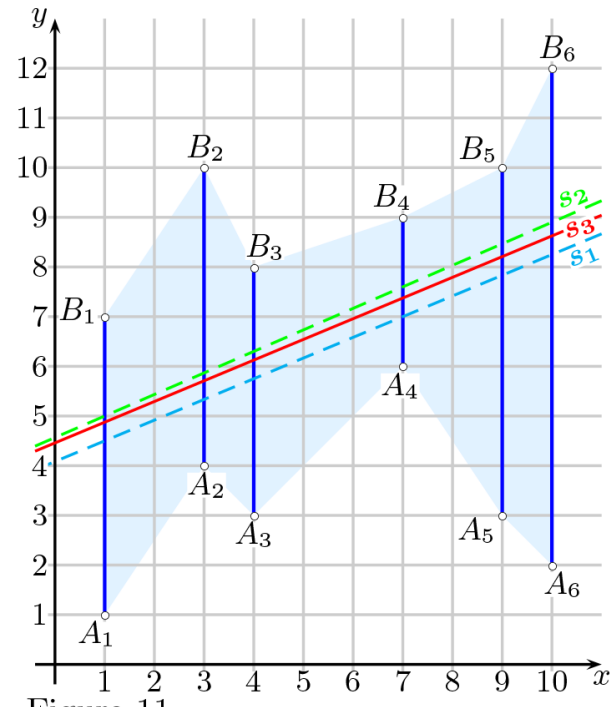

Figure 11.

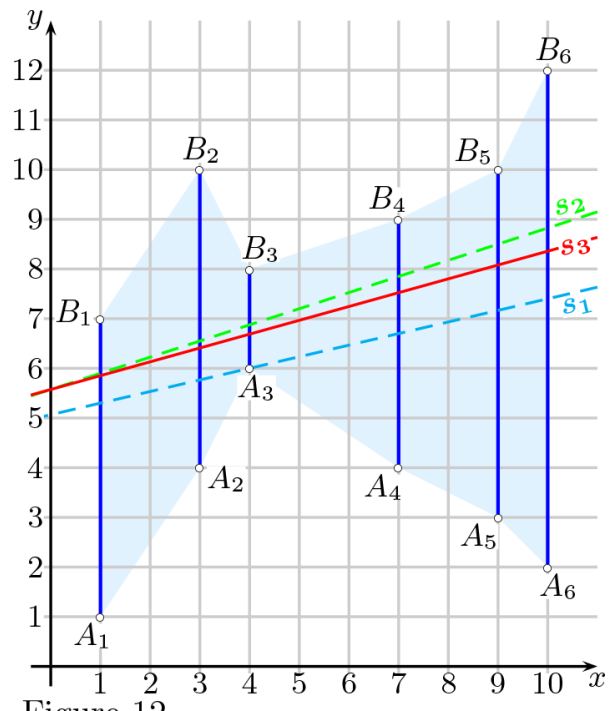

Figure 12.

We observe the polygon in Figure 8:

- $\quad$ the signed area $A$ of the polygon $P_{1} P_{2} P_{3} P_{4} P_{5}$ is $\left(P_{0} \equiv P_{5}\right)$ :

$$
\begin{aligned}
A=\frac{1}{2} & \sum_{i=0}^{4}\left(x_{P_{i}} y_{P_{i+1}}-x_{P_{i+1}} y_{P_{i}}\right)=\frac{1}{2}\left(1 \cdot \frac{5}{2}-\frac{1}{2} \cdot 1\right)+ \\
& +\frac{1}{2}\left(\frac{1}{2} \cdot \frac{43}{6}-\left(-\frac{1}{6}\right) \cdot \frac{5}{2}\right)+\frac{1}{2}\left(\left(-\frac{1}{6}\right) \cdot \frac{20}{3}-\frac{1}{3} \cdot \frac{43}{6}\right)+ \\
& +\frac{1}{2}\left(\frac{1}{3} \cdot \frac{11}{2}-\frac{1}{2} \cdot \frac{20}{3}\right)+\frac{1}{2}\left(\frac{1}{2} \cdot 1-1 \cdot \frac{11}{2}\right)= \\
=1 & +2-\frac{7}{4}-\frac{3}{4}-\frac{5}{2}=-2
\end{aligned}
$$

- $\quad$ and than we will find the centroid $C\left(m_{C}, b_{C}\right)$ of the polygon following the calculations:

$$
\begin{aligned}
m_{C} & =\frac{1}{6 A} \sum_{i=0}^{4}\left(x_{P_{i}}+x_{P_{i+1}}\right)\left(x_{P_{i}} y_{P_{i+1}}-x_{P_{i+1}} y_{P_{i}}\right)= \\
& =-\frac{1}{12}\left[\left(1+\frac{1}{2}\right) \cdot 2+\left(\frac{1}{2}-\frac{1}{6}\right) \cdot 4+\left(-\frac{1}{6}+\frac{1}{3}\right) \cdot\left(-\frac{7}{2}\right)+\right.
\end{aligned}
$$




$$
\begin{aligned}
& \left.+\left(\frac{1}{3}+\frac{1}{2}\right) \cdot\left(-\frac{3}{2}\right)+\left(\frac{1}{2}+1\right) \cdot(-5)\right]= \\
= & -\frac{1}{12}\left(\frac{3}{2} \cdot 2+\frac{1}{3} \cdot 4-\frac{1}{6} \cdot \frac{7}{2}-\frac{5}{6} \cdot \frac{3}{2}-\frac{3}{2} \cdot 5\right)= \\
= & -\frac{1}{12}\left(3+\frac{4}{3}-\frac{7}{12}-\frac{5}{4}-\frac{15}{2}\right)=\frac{\mathbf{5}}{\mathbf{1 2}}, \\
b_{C}= & \frac{1}{6 A} \sum_{i=0}\left(y_{P_{i}}+y_{P_{i+1}}\right)\left(x_{P_{i}} y_{P_{i+1}}-x_{P_{i+1}} y_{P_{i}}\right)= \\
= & -\frac{1}{12}\left[\left(1+\frac{5}{2}\right) \cdot 2+\left(\frac{5}{2}+\frac{43}{6}\right) \cdot 4+\left(\frac{43}{6}+\frac{20}{3}\right) \cdot\left(-\frac{7}{2}\right)+\right. \\
& \left.+\left(\frac{20}{3}+\frac{11}{2}\right) \cdot\left(-\frac{3}{2}\right)+\left(\frac{11}{2}+1\right) \cdot(-5)\right]= \\
= & \frac{1}{12}\left(\frac{7}{2} \cdot 2+\frac{29}{3} \cdot 4-\frac{83}{6} \cdot \frac{7}{2}-\frac{73}{6} \cdot \frac{3}{2}-\frac{13}{2} \cdot 5\right)= \\
= & \frac{1}{12}\left(7+\frac{116}{3}-\frac{581}{12}-\frac{73}{4}-\frac{65}{2}\right)=\frac{\mathbf{1 0 7}}{\mathbf{2 4}} ;
\end{aligned}
$$

- we get the straight line $s_{3}$ (Figure 11) with equation $y=\frac{5}{12} x+\frac{107}{24}$ that is associated with the centroid $C$.

Now we observe the polygon in Figure 10:

- $\quad$ the signed area $\mathrm{A}$ of the polygon $P_{1} P_{2} P_{3} P_{4}$ is $\left(P_{0} \equiv P_{4}\right)$ :

$$
\begin{aligned}
A=\frac{1}{2} & \sum_{i=0}^{3}\left(x_{P_{i}} y_{P_{i+1}}-x_{P_{i+1}} y_{P_{i}}\right)=\frac{1}{2}\left(\frac{1}{2} \cdot \frac{14}{5}-\frac{4}{5} \cdot \frac{11}{2}\right)+ \\
& +\frac{1}{2}\left(\frac{4}{5} \cdot \frac{22}{3}-\left(-\frac{1}{3}\right) \cdot \frac{14}{5}\right)+\frac{1}{2}\left(-\frac{1}{3} \cdot \frac{20}{3}-\frac{1}{3} \cdot \frac{22}{3}\right)+ \\
& +\frac{1}{2}\left(\frac{1}{3} \cdot \frac{11}{2}-\frac{1}{2} \cdot \frac{20}{3}\right)=-\frac{3}{2}+\frac{17}{5}-\frac{7}{3}-\frac{3}{4}=-\frac{\mathbf{7 1}}{\mathbf{6 0}}
\end{aligned}
$$

- $\quad$ we will find the centroid $C\left(m_{C}, b_{C}\right)$ of the polygon following the calculations: 


$$
\begin{aligned}
& m_{C}= \frac{1}{6 A} \sum_{i=0}^{4}\left(x_{P_{i}}+x_{P_{i+1}}\right)\left(x_{P_{i}} y_{P_{i+1}}-x_{P_{i+1}} y_{P_{i}}\right)= \\
&=-\frac{10}{71}\left[\left(\frac{1}{2}+\frac{4}{5}\right) \cdot(-3)+\left(\frac{4}{5}-\frac{1}{3}\right) \cdot \frac{34}{5}+\right. \\
&\left.\quad+\left(-\frac{1}{3}+\frac{1}{3}\right) \cdot\left(-\frac{14}{3}\right)+\left(\frac{1}{3}+\frac{1}{2}\right) \cdot\left(-\frac{3}{2}\right)\right]= \\
&=-\frac{10}{71}\left(-\frac{39}{10}+\frac{238}{75}+0-\frac{5}{4}\right)=\frac{\mathbf{5 9 3}}{\mathbf{2 1 3 0}}, \\
& b_{C}=\frac{1}{6 A} \sum_{i=0}^{4}\left(y P_{i}+y_{P_{i+1}}\right)\left(x_{P_{i}} y_{P_{i+1}}-x_{P_{i+1}} y_{P_{i}}\right)= \\
&=-\frac{10}{71}\left[\left(\frac{11}{2}+\frac{14}{5}\right) \cdot(-3)+\left(\frac{14}{5}+\frac{22}{3}\right) \cdot \frac{34}{5}+\right. \\
&\left.+\left(\frac{22}{3}+\frac{20}{3}\right) \cdot\left(-\frac{14}{3}\right)+\left(\frac{20}{3}+\frac{11}{2}\right) \cdot\left(-\frac{3}{2}\right)\right]= \\
&=- \frac{10}{71}\left(\frac{83}{10}+\frac{152}{15}+0-\frac{5}{4}\right)=\frac{\mathbf{1 1 8 7 3}}{\mathbf{2 1 3 0}} ;
\end{aligned}
$$

- we get the straight line $s_{3}$ (Figure 12) with equation $y=\frac{593}{2130} x+\frac{11873}{2130}$ that is associated with the centroid $C$.

In the two examples it seems clear that the straight line that we find with the Algorithm 1 is not so "good", while it seems that the two centroids give us two straight lines quite similar. If this fact would be always true, then we might consider more appropriate to use the Algorithm 2, at least to avoid the huge amount of calculations that must be done to find the straight line with the Algorithm 3. But this result is accidental, due to the few examples seen, and the next example is the confirmation. I end this article proposing such an example without calculations.

In Figure 13 and 14 we have:

$$
\begin{array}{llll}
A_{1} A_{2}: y=x, & P_{1}(1,0), & B_{1} B_{4}: y=11, & P_{5}(0,11), \\
A_{2} A_{3}: y=\frac{1}{2} x+1, & P_{2}\left(\frac{1}{2}, 1\right), & B_{4} B_{5}: y=\frac{1}{2} x+8, & P_{6}\left(\frac{1}{2}, 8\right), \\
A_{3} A_{6}: y=3, & P_{3}(0,3), & B_{5} B_{6}: y=x+4, & P_{7}(1,4), \\
A_{6} B_{1}: y=-x+12, & P_{4}(-1,12), & B_{6} A_{1}: y=\frac{3}{2} x-\frac{1}{2}, & P_{8}\left(\frac{3}{2},-\frac{1}{2}\right) .
\end{array}
$$




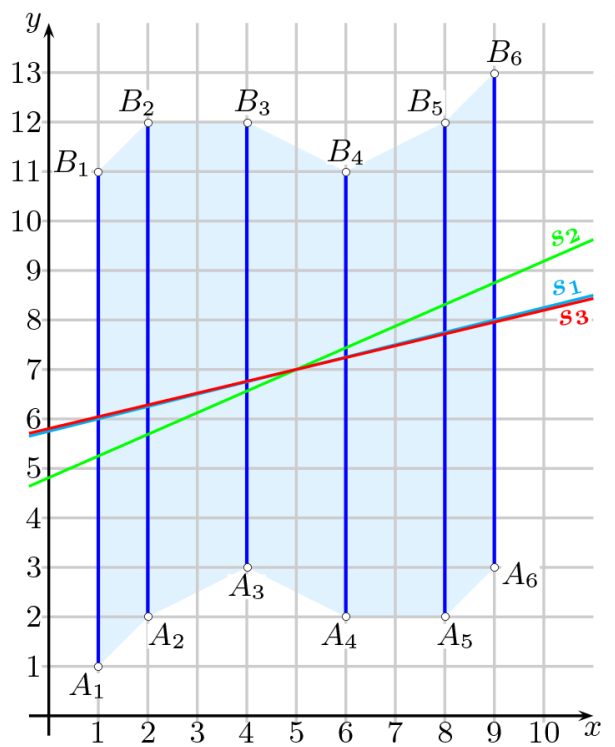

Figure 13.

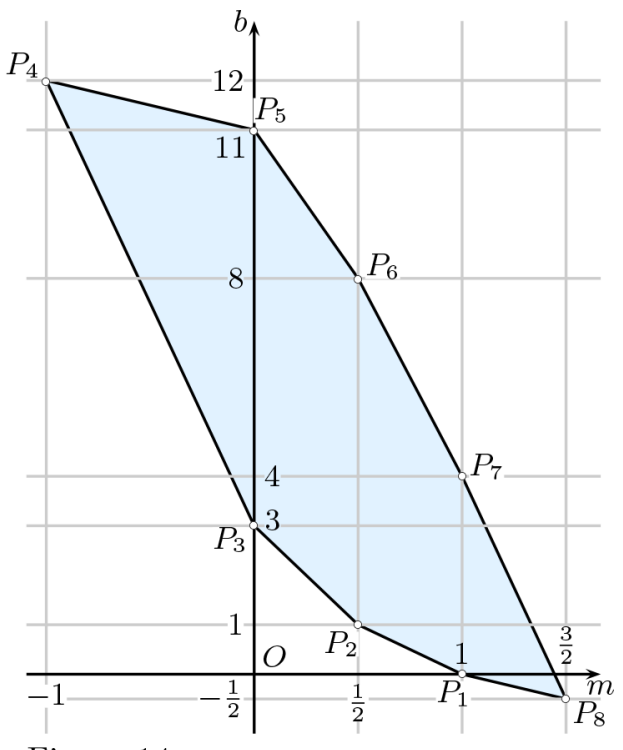

Figure 14.

$$
s_{1}: y=\frac{1}{4} x+\frac{23}{4}, \quad s_{2}: y=\frac{7}{16} x+\frac{77}{16}, \quad s_{3}: y=\frac{11}{46} x+\frac{267}{46} .
$$

The latest example is a further confirmation of what we thought, that is that the line that we find with Algorithm 3 seems to be "better" than others. At this point it would be interesting to study the set of lines in $\mathscr{L}\left(\mathbb{R}^{2}\right)$ with a different point of view, perhaps less geometrical and a little bit more statistical, considering that now, as we have seen with Theorem 9, there's a way to know if the segments are intersected by a straight line. At the end we want to remember having had the opportunity to consult with articles [1] and [2] which, with different purposes and in different ways, deal with a topic very close to the one discussed in this article.

And finally we will be grateful to Omar Lakkis for the helpful suggestions he gave us.

\section{BIBLIOGRAPHY}

1. H. Edelsbrunner, Finding Transversals for Sets of Simple Geometric Fig- ures, Theoretical Computer Science 35 (1985), 55-69.

2. H. Edelsbrunner, H. A. Maurer, F. P. Preparata, A. L. Rosenberg, E. Welzl and D. Wood, Stabbing Line Segments, BIT Numerical Mathematics 22 (1982), 274-281 